\title{
A global analytical molecular pharmacological study of the endocrinological pharmacotherapeutic rationale of anti-diabetic prescriptions appraisal attributes for metformin and sitagliptin, with evaluation of anti-diabetic tertiary medical healthcare patient satisfaction
}

\author{
Moumita Hazra ${ }^{1-4 *}$ \\ ${ }^{1}$ Department of Pharmacology, Mamata Medical College and Hospitals, Khammam, Telangana, India \\ ${ }^{2}$ Department of Pharmacology, Rama Medical College Hospital and Research Centre, Kanpur, Uttar Pradesh, India \\ ${ }^{3}$ Medical Director, Medical Superintendent, Laboratory Director, Medical Academics and Research Director, Consultant \\ Clinical Pharmacological Physician, Consultant Clinical Pathologist, Consultant Drug Quality and Safety Physician, Pharmaco- \\ Haemo-Materio-Vigilance Specialist, Dr. Moumita Hazra's Polyclinic and Diagnostic Centre, Dr. Moumita Hazra's Academic \\ Centre, Dr. Moumita Hazra's Educational Centre, Hazra Nursing Home, Howrah, Kolkata, West Bengal, India, World; \\ ${ }^{4}$ Former Assistant Medical Director, GIOSTAR IRM Institutes, Hospitals and Laboratories, New Delhi, India, USA, World
}

Received: 04 October 2021

Revised: 13 November 2021

Accepted: 15 November 2021

*Correspondence:

Dr. Moumita Hazra,

Email: drmoumitahazra.198017thjune@gmail.com

Copyright: (C) the author(s), publisher and licensee Medip Academy. This is an open-access article distributed under the terms of the Creative Commons Attribution Non-Commercial License, which permits unrestricted non-commercial use, distribution, and reproduction in any medium, provided the original work is properly cited.

\section{ABSTRACT}

Background: The inhibition of dipeptidyl peptidase- 4 by anti-diabetic drugs dipeptidyl peptidase- 4 inhibitors enhances hormonal activity of incretins (GLP-1, GIP, GRP), stimulates insulin release and reduces glucagon secretion, producing anti-hyperglycaemic activity among type II diabetics. The objective of this study was a global analytical molecular pharmacological study of the endocrinological rationale of anti-diabetic prescriptions appraisal attributes for metformin and sitagliptin, along with anti-diabetic tertiary medical healthcare patient satisfaction evaluation.

Methods: 100 new early moderate grade type II diabetics were prescribed oral metformin $500 \mathrm{mg}$ or sitagliptin $25 \mathrm{mg}$ once daily for 3 months, in monotherapy, or in combination therapy, or in a mixed regimen of monotherapy and combination therapy. The patients' endocrinological pharmacotherapeutic compliance was analysed. The number of prescriptions for metformin and sitagliptin was recorded; and prescription percentages were calculated. The completeness and molecular basis of prescription content attributes were analysed. The molecular basis of antidiabetic pharmacotherapeutics, was analysed. The anti-diabetic tertiary medical healthcare patient satisfaction was evaluated by patient response to different attributes of anti-diabetic treatment.

Results: All the patients had completed the study, with no adverse effects related drop-out, lost to follow-up or voluntarily withdrawn patients. The prescription rates of metformin was $75 \%$ (75 prescriptions), followed by sitagliptin: $25 \%$ (25 prescriptions).100\% prescriptions were complete for each prescription content attribute. The molecular pharmacotherapeutic response mechanisms were significantly efficacious. All the patients were satisfied with each anti-diabetic medical healthcare attribute.

Conclusions: The patient endocrinological pharmacotherapeutic compliance was significantly high. Metformin was most commonly prescribed, followed by sitagliptin. The prescription content analyses showed $100 \%$ completeness, with significant pharmacotherapeutic molecular efficacy. There was ample anti-diabetic medical healthcare satisfaction.

Keywords: Biguanides, Dipeptidyl peptidase-4 inhibitors, Prescription's appraisal, Patient compliance, Molecular Pharmacology, Anti-diabetic medical healthcare patient satisfaction 


\section{INTRODUCTION}

According to the American Association of Clinical Endocrinologists (AACE) guidelines for type II diabetes mellitus management, lifestyle therapy, medically assisted weight loss, and individual goals of achieving haemoglobin A1c (HbAlc) level of $\leq 6.5 \%$ are quite significant, for a comprehensive antidiabetic therapeutic regimen. The varied patient characteristics, like glycaemic index and weight, lifestyle, co-morbidities, and undesirable side effects of pharmaco-therapeutic management, ascertain the choice of precise antidiabetic pharmacotherapeutic agents. The adverse effects that commonly manifest with orally administered antidiabetic drugs include hypoglycaemia, weight gain due to hyperinsulinemia, gastrointestinal symptoms, and hepatorenal toxicity. An increase, observed in the occurrence of these adverse effects, demands much safer antidiabetic drugs. And, this re-search for the safer antidiabetic drugs, retains under consideration some critical effects like the drug's potential for hypo-glycaemia, weight gain, and long term side effects. ${ }^{1-4}$

Metformin, has fairly effective outcomes, as a monotherapeutic as well as a combination anti-diabetic drug, while overcoming insulin resistance and lowering serum glucose levels, by the activation of 5 ' adenosine monophosphate (AMP) activated protein kinase. Metformin is efficacious, safe, inexpensive, and may reduce the risk of cardiovascular co-morbid events and mortality. It has beneficial effects in $\mathrm{HbA1c}$ and weight reduction. ${ }^{1-3,5}$ Inhibition of the enzyme dipeptidyl peptidase-4 (DPP-4) by dipeptidyl peptidase-4 inhibitors enhances the hormonal activity of incretins, like glucagon like peptide-1 and other bioactive peptides (glucosedependent insulinotropic polypeptide, gastrin releasing peptide), thus stimulating the release of insulin and reducing the secretion of glucagon, when given in monotherapy or in combination with metformin. This pharmacodynamic effect decreases the blood glucose levels as well as HbA1c levels among type II diabetic patients, without causing severe hypoglycaemia. While anti-dipeptidyl peptidase-4 drugs have wide-ranged effects on antihyperglycemic conditions, there has been an overall dirth of studies regarding the role of these drugs on beta-cell function, peripheral insulin sensitivity, insulin resistance and serum GLP-1 concentrations, in

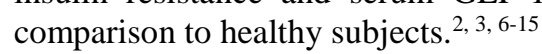

\section{Objectives}

The objectives of this analytical molecular pharmacological study was to analyse the endocrinological rationale of the various features of antidiabetic prescriptions appraisal for metformin and sitagliptin, along with an evaluation of the anti-diabetic medical healthcare patient satisfaction, in global tertiary care medical colleges, hospitals, nursing homes, polyclinics and diagnostic centres.

\section{METHODS}

At first, the Institutional Ethics Committee clearance and approval was taken for conducting this study. Then, this study was conducted in accordance with the ethical principles of Declaration of Helsinki and Good Clinical Practices contained within the International Council for Harmonization of Technical Requirements for Pharmaceuticals for Human Use (ICH-E6), and in compliance with the global regulatory requirements. An informed consent was obtained from each patient. Current study was conducted in accordance with the ethical principles of declaration of Helsinki and good clinical practices contained within the international council for harmonization of technical requirements for pharmaceuticals for human use (ICH-E6), and in compliance with the global regulatory requirements. An informed consent was obtained from each patient.

\section{Inclusion criteria}

The inclusion criteria for this study were as follows: patients of any gender, patients within 35 and 60 years, patients presenting with new type II diabetes mellitus, of early moderate grade, type II diabetes mellitus American Diabetes Association diagnosis criteria, co-operative and conscious patients, patients willing to undergo all pre and post- treatment investigations and willing to complete the entire course of treatment, patients who have given consent and are willing to go for a follow-up, patients not taking any previous anti-diabetic drug and patients not taking any concomitant medication.

\section{Exclusion criteria}

The exclusion criteria for this study were as follows: uncooperative or unconscious patients, patients below 35 and above 60 years, patients presenting with any grade other than early moderate grade of diabetes, patients with a history of hypersensitivity to any of the study drugs, patients with high risk diseases or co-morbidities, cardiac, renal or any other associated complications or co-morbidities, any chronic disease intervening with the study data, pregnant or lactating women, paediatric or geriatric patients, other associated medical illness or disorders, having impact on study results and female patients using hormonal contraceptives.

\section{Study type}

A global, multi-centre, prospective, analytical study of the clinical prescription's appraisal with an evaluation of the anti-diabetic medical healthcare patient satisfaction was conducted.

\section{Study population}

The study population consisted of 100 treated new type II diabetes mellitus patients, of early moderate grade, in 
global tertiary care medical colleges, hospitals, nursing homes, polyclinics and diagnostic centres.

\section{Study period}

The study period, comprising of the periods for the research study and the compilation of the study literature, was 5 months, from July 2021 to December 2021.

\section{Place of Study}

This research study and the compilation of the study literature was done in the Departments of Pharmacology, Clinical Pharmacology, Molecular Pharmacology, Pharmacovigilance, Rational Pharmacotherapeutics, Evidence Based Medicine, Internal Medicine, Endocrinology, Pathology, Clinical Pathology, and Molecular Diagnostics, in Dr. Moumita Hazra's Polyclinic And Diagnostic Centre, Dr. Moumita Hazra's Academic Centre, Dr. Moumita Hazra's Educational Centre, Hazra Nursing Home, Rama Medical College Hospital and Research Centre, Mamata Medical College and Hospitals, and GIOSTAR IRM Institutes, Hospitals and Laboratories.

\section{Study procedure}

100 new early moderate grade type II diabetes mellitus patients, were prescribed oral metformin $500 \mathrm{mg}$ once daily, or sitagliptin $25 \mathrm{mg}$ once daily, for 3 months, in monotherapy, or in combination therapy, or in a mixed regimen of monotherapy and combination therapy. The patients' characteristics, diabetic symptoms assessment, patients' disease and disease-related history were recorded with a study proforma. Then, thorough general physical examination and systemic examination were performed on the patients under study. The relevant blood, urine and other investigations were done to confirm the progressing health status of the patients being treated. The efficacy assessment was done, by recording the fasting and the post-prandial blood sugar level, HbA1c level and urine routine examination findings including sugar and albumin levels and microscopy, at subsequent intervals, and follow-up. The safety assessment was done by the monitoring of adverse drug reactions, at subsequent intervals, and follow-up.

The patient response based on their endocrinological pharmacotherapeutic compliance was thoroughly analysed, by deriving the statistical percentages of the total patients who participated in the study, total patients who completed the study, total patients who were lost to follow-up, total patients who had dropped out due to adverse effects, and total patients who had withdrawn voluntarily, to determine the patient compliance to antidiabetic drugs.

The prescription patterns of both the anti-diabetic drugs were analysed. The number of prescriptions of 100 patients treated with each drug: metformin and sitagliptin was recorded; and the percentage of prescriptions for each drug was calculated. The prescription content analysis, of all the 100 prescriptions, was done. The different aspects of the prescription contents, like the completeness of the prescription contents, the dose of drug, the duration of treatment, the instructions of medication, the frequency of drug intake, the name of the drug and the dosage form of the drug were thoroughly analysed and recorded, and the various derived observations were statistically analysed as the prescription content analysis percentages. The endocrinological rationale of the various attributes of these anti-diabetic prescriptions content for metformin and sitagliptin, were thoroughly appraised and analysed. In correlation, the molecular pharmacological basis of these anti-diabetic pharmacotherapeutics, like metformin and sitagliptin, were also thoroughly analysed from wide ranged molecular pharmacological research, review and case presentation study literature to derive the endocrinological rationale of the clinical pharmacotherapeutic use of anti-diabetic drugs, like biguanides and dipeptidyl peptidase- 4 inhibitors, among type II diabetes mellitus patients, belonging to a widely multivariate pharmacoepidemiologic, pharmacogenomic and epigenetic population, spanning a pan Asian as well as a pan non-Asian pharmacogeographical distribution.

The anti-diabetic medical healthcare patient satisfaction was evaluated by the response of the patients to the different attributes, like immediate treatment delivery, appropriate and convenient investigations and treatment, quickly controlled diabetes, safe and tolerable treatment, easily accessible medications, convenient administration of medications, and maintenance of symptom-free controlled diabetic period. These observations were recorded and thoroughly analysed.

\section{Statistical analysis}

The prescription contents evaluation and patient compliance assessment were performed by different types of statistical analyses in percentages. These research study results were further depicted by graphical illustrations.

\section{RESULTS}

The demographic characteristics of the patients were comparable. 100 new type II diabetes mellitus patients, of early moderate grade, receiving metformin or sitagliptin monotherapy, or combination therapy, or mixed regimen of monotherapy and combination therapy, for 3 months, all the patients had completed the study thoroughly, with no adverse effects related drop-out patients, lost to follow-up patients or voluntarily withdrawn patients (Figure 1). Metformin was most commonly prescribed (75 prescriptions, $75 \%$ ), followed by sitagliptin (25 prescriptions, 25\%) (Figure 2). 


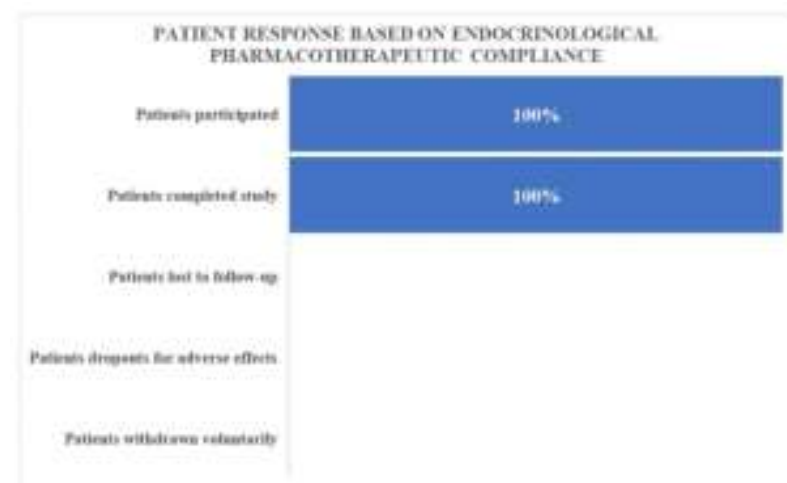

Figure 1: Patient response based on endocrinological pharmacotherapeutic compliance.

The prescription rates of anti-diabetic drugs were as follows: metformin>sitagliptin. The completeness of the prescription contents, the dose of drug, the duration of treatment, the instructions of medication, the frequency of drug intake, the name of the drug and the dosage form of the drug were found in $100 \%$ of prescriptions (Table 1).

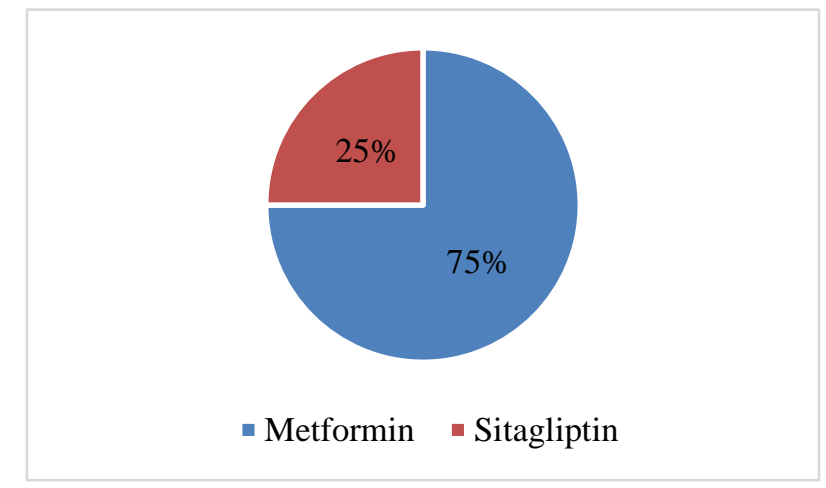

Figure 2: The prescription rates of anti-diabetic drugs in percentages.

Table 1: Prescription content analysis for different anti-diabetic drugs.

\begin{tabular}{|ll|}
\hline $\begin{array}{l}\text { Prescription contents } \\
\text { Completeness of prescription } \\
\text { contents }\end{array}$ & Results N (\%) \\
\hline Dose of drug & $100(100)$ \\
\hline Duration of treatment & $100(100)$ \\
\hline Instruction of medication & $100(100)$ \\
\hline Frequency of drug intake & $100(100)$ \\
\hline Name of drug & $100(100)$ \\
\hline Dosage form of the drug & $100(100)$ \\
\hline
\end{tabular}

The monotherapy, or combination therapy, or mixed regimen of monotherapy and combination therapy of metformin or sitagliptin, was observed to be quite efficacious, which had controlled type II diabetes mellitus among new patients, with significant decrease in the blood sugar levels and the HbAlc levels, in the successive 3 months. The adverse effects observed with monotherapy, or combination therapy, or mixed regimen of monotherapy and combination therapy, was statistically non-significant. Therefore, the monotherapy, or combination therapy, or mixed regimen of monotherapy and combination therapy, were safe and tolerable.

The correlated molecular pharmacological analysis established an appropriate and adequate clinical pharmacotherapeutic application of metformin and sitagliptin among type II diabetic patients, based on the analytical evidence-based deductions regarding the systematic structural and organisational synchrony in the endocrinological pharmacodynamic and pharmacotherapeutic response mechanisms of metformin and sitagliptin, which were significantly effective in the comprehensive anti-diabetic treatment.

All the global patients were satisfied with the different attributes of anti-diabetic tertiary medical healthcare provided, like immediate treatment delivery, appropriate convenient investigations and treatment, quickly controlled diabetes, safe and tolerable treatment, early accessible medications, convenient administration of medications and maintenance of symptom-free controlled diabetic period (Table 2).

\section{DISCUSSION}

Diabetes mellitus type II (T2D), a chronic metabolic disorder, has sharply increased on a global scale, in the recent times. According to the International Diabetes Federation (IDF), there were 415 million patients diagnosed with T2D. Diabetes among Asian populations can be differentiated from non-Asian populations in the world, by the early decrease in beta-cell function resulting in high postprandial blood glucose and the development to chronic diabetic complications occurring at an early stage of the disease. Hence, a pharmacotherapeutic agent who increases the beta-cell function plays an important role in antihyperglycemic protocols.

Presently, anti-DPP4 antihyperglycemic drugs have been widely used for patients with T2D under the guidelines of diabetes associations. These drugs possess proven effectiveness in the improvement of the beta-cell function, thereby ameliorating serum incretin hormone concentrations (two major incretins, GLP-1 and glucosedependent insulinotropic polypeptide, GIP) - an anti-betacell apoptosis agent. The two existing incretin-related therapies for patients with T2D, are glucagon-like peptide-1 agonists, like exedin-4 and dipeptidyl peptidase-IV inhibitor, like sitagliptin. Incretin therapies, as the first-line drug for newly diagnosed patients with $\mathrm{T} 2 \mathrm{D}$, in monotherapy or in combination with other antidiabetic drugs such as biguanide, sulfonylurea, or insulin, according to the American association of clinical endocrinologists (AACE) issued guidelines for 
antihyperglycemic treatment protocol, has remained highly effective, not only in glycaemic control, but also in the prevention from chronic diabetic complications, as well.

GLP-1 is a potent insulin secretagogue which exhibits glucose dependent insulin secretion. In few in vitro studies, GLP-1 was found to be capable of healing the age-related reduction in the beta-cell function that occurred due to the following reasons: recruitment of beta-cells into a secretory mode; activation of the gene for glucose sensitivity of beta-cells; and reduction of beta-cell apoptosis. The therapeutic approaches on the old Wistar rats with GLP-1 led to normal insulin secretion, along with the increases of beta-cell mass and pancreas cell proliferation. It was also hypothesized that besides the hypoglycaemic effect of anti-DPP4 drugs, it may be the increase of GLP-1 that ultimately resulted in the increase of beta-cell functions.

In another study, serum GLP-1 concentrations accelerated rapidly after treatment and the regression analysis confirmed that serum GLP-1 concentrations were independent variable causing the amelioration of insulin sensitivity and insulin resistance. This study has shown that there were improvements in beta cell function, yet there were 4 patients who had still shown low beta cell functions in comparison to those in the control group. . $^{2,3,6}$

Table 2: Anti-diabetic tertiary medical healthcare patient satisfaction.

\begin{tabular}{|c|c|c|c|}
\hline Healthcare attributes & $\begin{array}{l}\text { Patients' response: } \\
\text { Satisfied }\end{array}$ & $\begin{array}{l}\text { Patients' response: } \\
\text { indeterminate }\end{array}$ & $\begin{array}{l}\text { Patients' response: } \\
\text { unsatisfied }\end{array}$ \\
\hline Immediate treatment delivery & Satisfied & - & - \\
\hline $\begin{array}{l}\text { Appropriate and convenient } \\
\text { investigations and treatment }\end{array}$ & Satisfied & - & - \\
\hline Quickly controlled diabetes & Satisfied & - & - \\
\hline Safe and tolerable treatment & Satisfied & - & - \\
\hline Easily accessible medications & Satisfied & - & - \\
\hline Convenient administration of medications & Satisfied & - & - \\
\hline $\begin{array}{l}\text { Maintenance of symptom-free controlled } \\
\text { diabetic period }\end{array}$ & Satisfied & - & - \\
\hline
\end{tabular}

Recent studies have suggested that SARS-CoV-2 may also bind dipeptidyl peptidase 4 (DPP-4 or CD26) when entering the cells of the respiratory tract. Based on the modelling of SARS-CoV-2 structure and receptors, it has been also postulated that DPP-4 may facilitate the SARSCoV-2 entry into the target cells, due to its high homology with Middle East respiratory syndrome coronavirus. The molecular interaction between the SARS-CoV-2 spike glycoprotein S1 and human DPP-4 may represent a potential factor that promotes SARSCoV-2 hijacking and virulence. Therefore, inhibiting this interaction may have the potential to improve clinical outcomes of COVID-19. Oral sitagliptin, a highly selective DPP-4 inhibitor having glucose-lowering effects, along with immunoregulatory and antiinflammatory effects, is approved by the food and drug administration, for its efficacy in increasing the bioavailability of glucagon-like peptide 1 (GLP-1). Sitagliptin has been shown to have the potential to inhibit hepatitis $\mathrm{C}$ virus replication, to suppress chemokine release, and to reduce interleukin-6 production. Sitagliptin has also showed high selectivity for DPP-4 and may have favourable effects on B-cells by exerting an anti-inflammatory function. Owing to all these pharmaco-molecular and pharmaco-dynamic mechanisms, DPP-4 inhibitors have been suggested to be of potential benefit for patients with COVID-19, particularly with the co-morbidities of type 2 diabetes. Although type 2 diabetes does not enhance the susceptibility to SARS-CoV-2 infection, type 2 diabetes has been associated with the worse outcomes of COVID19. Type 2 diabetes also increases the mortality risk in patients with COVID-19, particularly in those with more severe disease. In patients with type 2 diabetes and COVID-19, poorly controlled blood glucose levels are associated with markedly higher mortality as compared with subjects with better metabolic control. , $3,7-15^{-15}$

In this study, the demographic characteristics of the patients were comparable. The patient response based on

endocrinological pharmacotherapeutic compliance showed that among 100 new type II diabetes mellitus patients, of early moderate grade, receiving metformin or sitagliptin monotherapy, or combination therapy, or mixed regimen of monotherapy and combination therapy, for 3 months, all the patients had completed the study thoroughly, with no adverse effects related drop-out patients, lost to follow-up patients or voluntarily withdrawn patients. The prescription rates of different anti-diabetic drugs in percentages showed that metformin was most commonly prescribed (75 prescriptions, $75 \%$ ), followed by sitagliptin (25 prescriptions, 25\%). The prescription rates of anti-diabetic drugs were as follows: metformin>sitagliptin. The completeness of the prescription contents, the dose of drug, the duration of treatment, the instructions of medication, the frequency of drug intake, the name of the drug and the dosage form 
of the drug were found in $100 \%$ of prescriptions. The correlated molecular pharmacological analysis established an appropriate and adequate clinical pharmacotherapeutic application of metformin and sitagliptin among type II diabetic patients, based on the analytical evidence-based deductions regarding the systematic structural and organisational synchrony in the endocrinological pharmacodynamic and pharmacotherapeutic response mechanisms of metformin and sitagliptin, which were significantly effective in the comprehensive anti-diabetic treatment. The monotherapy, or combination therapy, or mixed regimen of monotherapy and combination therapy of metformin or sitagliptin, was observed to be quite efficacious, which had controlled type II diabetes mellitus among new patients, with significant decrease in the blood sugar levels and the HbA1c levels, in the successive 3 months. The adverse effects observed with monotherapy, or combination therapies, or mixed regimen of monotherapy and combination therapy, were statistically nonsignificant. Therefore, the monotherapy, or combination therapy, or mixed regimen of monotherapy and combination therapy, were safe and tolerable. The patients were satisfied with the anti-diabetic tertiary medical healthcare provided. This study did not have any significant limitation.

In a similar study, the demographic characteristics of the patients were comparable. Metformin was most commonly prescribed (120 prescriptions, 80\%) followed by sitagliptin (21 prescriptions, 14\%) and remogliflozin (9 prescriptions, 6\%). The prescription rates of antidiabetic drugs were as follows: metformin>sitagliptin> remogliflozin. The monotherapy, or combination therapy, or mixed regimen of monotherapy and combination therapy of metformin, sitagliptin, or gemigliptin, was observed to be quite efficacious, which had controlled T2DM among new patients, with significant decrease in the blood sugar levels and the HbAlc levels, in the successive 3 months. The adverse effects observed with monotherapy, or combination therapy, or mixed regimen of monotherapy and combination therapy, were statistically non-significant. Therefore, the monotherapy, or combination therapy, or mixed regimen of monotherapy and combination therapy, was safe and tolerable. ${ }^{1}$

In another study, among 150 new type II diabetes mellitus patients, of early moderate grade, receiving metformin monotherapy for 1 month, 50 uncontrolled diabetic patients, who had achieved adequate glycaemic control with metformin monotherapy, or who were lost to follow-up, or who had dropped out due to adverse effects, or who had withdrawn voluntarily, were excluded from the study. The remaining 100 patients, received remogliflozin and metformin combination therapy, for 15 days. These patients had completed the study thoroughly, with no adverse effects related drop-out patients, lost to follow-up patients or voluntarily withdrawn patients. The demographic characteristics of the patients were comparable. The monotherapy of metformin and the combination therapy of remogliflozin and metformin were observed to be safe, which had controlled type II diabetes mellitus among new patients, with significant decrease in the blood sugar levels and the HbAlc levels, in 1.5 months. There were no adverse effects observed with the monotherapy of metformin as well as the combination therapy of remogliflozin and metformin, which were statistically non-significant. The monotherapy of metformin and the combination therapy of remogliflozin and metformin were observed to be safe and tolerable. ${ }^{2}$

In yet another study, the demographic characteristics of the patients were comparable. Metformin was most commonly prescribed ( 80 prescriptions, $80 \%$ ), followed by sitagliptin (16 prescriptions, 16\%), and gemigliptin (4 prescriptions, $4 \%$ ). The prescription rates of anti-diabetic drugs were as follows: metformin>sitagliptin> gemigliptin. The monotherapy, or combination therapy, or mixed regimen of monotherapy and combination therapy of metformin, sitagliptin or gemigliptin, was observed to be quite efficacious, which had controlled type II diabetes mellitus among new patients, with significant decrease in the blood sugar levels and the HbA1c levels, in the successive 3 months. The adverse effects observed with monotherapy, or combination therapy, or mixed regimen of monotherapy and combination therapy, was statistically non-significant. Therefore, the monotherapy, or combination therapy, or mixed regimen of monotherapy and combination therapy, were safe and tolerable. ${ }^{3}$

\section{CONCLUSION}

The patient response based on the endocrinological pharmacotherapeutic compliance was significantly high. The prescription rates of different anti-diabetic drugs in percentages were $75 \%$ of metformin prescriptions, followed by $25 \%$ of sitagliptin prescriptions; metformin being most commonly prescribed. The prescription content analyses showed $100 \%$ completeness, with significant pharmacotherapeutic molecular efficacy. There was ample global anti-diabetic patient healthcare satisfaction. Therefore, this analytical molecular pharmacological study delineates the endocrinological pharmacotherapeutic rationale of anti-diabetic prescriptions for metformin and sitagliptin, with utmost clarity, along with the presentation of a comprehensive anti-diabetic medical healthcare patient satisfaction, among the type II diabetic patients, in global tertiary care medical colleges, hospitals, nursing homes, polyclinics and diagnostic centres.

\section{ACKNOWLEDGEMENTS}

My profound gratitude to the Departments of Pharmacology, Clinical Pharmacology, Molecular Pharmacology, Pharmacovigilance, Rational Pharmacotherapeutics, Evidence Based Medicine, 
Internal Medicine, Endocrinology, Pathology, Clinical Pathology, and Molecular Diagnostics, in Dr. Moumita Hazra's Polyclinic And Diagnostic Centre, Dr. Moumita Hazra's Academic Centre, Dr. Moumita Hazra's Educational Centre, Hazra Nursing Home, Rama Medical College Hospital and Research Centre, Mamata Medical College and Hospitals, and GIOSTAR IRM Institutes, Hospitals and Laboratories, where this research study and the compilation of this study literature was performed, for the successful completion of this research project.

Funding: No funding sources

Conflict of interest: None declared

Ethical approval: The study was approved by the Institutional Ethics Committee

\section{REFERENCES}

1. Hazra M. An endocrinological pharmacovigilance study on the safety assessment of metformin monotherapy and the combination therapy of remogliflozin with metformin, in the new type II diabetes mellitus patients, in tertiary care medical college hospitals. World J Pharm Res 2021;10(11): 1443-53.

2. Hazra M. A study of the usual clinical pharmacological prescription patterns of metformin, Sitagliptin, and Remogliflozin among the early moderate grade new type ii diabetes mellitus patients in tertiary care hospitals. Int J Sci Stud 2021;9(5):1-4.

3. Hazra M. A clinical pharmacological study of the prevalent prescription patterns of metformin, sitagliptin and gemigliptin among the early moderate grade new type II diabetes mellitus patients in global tertiary care hospitals. Int J Pharm Sci Rev Res 2021; 70(1):180-4.

4. Dharmalingam M, Aravind SR, Thacker H, Paramesh S, Mohan B, Chawla M, et al. efficacy and safety of remogliflozin etabonate, a new sodium glucose cotransporter- 2 inhibitor, in patients with type 2 diabetes mellitus: A 24 week, randomized, double-blind, active controlled trial. Drugs. 2020;80:587-600.

5. American diabetes association. Pharmacologic approaches to glycaemic treatment: standards of medical care in diabetes-2021. Diabetes Care. 2021; 44 (Suppl. 1):S111-24.

6. Le TD, Nguyen NTP, Nguyen ST, Tran HTT, Nguyen LTH, Duong HH, et al. Sitagliptin increases beta-cell function and decreases insulin resistance in newly diagnosed vietnamese patients with type 2 diabetes mellitus. Diabetes Metab Syndr Obes. 2020;13:211927.

7. Solerte SB, D'Addio F, Trevisan R, Lovati E, Rossi A, Pastore I, et al. Sitagliptin treatment at the time of hospitalization was associated with reduced mortality in patients with type 2 diabetes and COVID-19: a multicenter, case-control, retrospective, observational study. Diabetes care. 2020;43:2999-3006.

8. LaMoia TE, Shulman GI. Cellular and molecular mechanisms of metformin action. Endocr Rev. 2021; 42:77-96.

9. Hussey EK, Kapur A, O’Connor-Semmes R, Tao W, Rafferty B, Polli JW, et al. Safety, pharmacokinetics and pharmacodynamicsof remogliflozin etabonate, a novel SGLT2 inhibitor, and metformin when coadministeredin subjects with type 2 diabetes mellitus. BMC Pharmacol Toxicol. 2013;14:25.

10. Zaccardi F, Jacquot E, Cortese V, Tyrer F, Seidu S, Davies MJ, et al. Comparative effectiveness of gliclazide modified release versussitagliptin as second-line treatment after metformin monotherapy in patients with uncontrolled type 2 diabetes. Diabetes Obes Metab. 2020;22:2417-26.

11. Garber AJ, Abrahamson MJ, Barzilay JI, Blonde L, Bloomgarden ZT, Bush MA, et al. Consensus statement by the American association of clinical endocrinologists and american college of endocrinology on the comprehensive type 2 diabetes management algorithm 2016 executive summary. Endocr Pract. 2016;22:84-113.

12. Inzucchi SE, Bergenstal RM, Buse JB, Diamant M, Ferrannini E, Nauck $M$, et al. Management of hyperglycaemia in type 2 diabetes, 2015: a patientcentred approach. Update to a position statement of the American diabetes association and the European Association for the study of diabetes. Diabetologia. 2015;58:429-42.

13. Tewarya S, Lucasa ES, Fujiharaa R, Kimanid PK, Polanco A, Brightona PJ. Impact of sitagliptin on endometrial mesenchymal stem-like progenitor cells: a randomised, double-blind placebo-controlled feasibility trial. EBio Medicine. 2020;51:1-10.

14. Raji A, Xu ZJ, Lam RLH, O’Neill EA, Kaufman KD, Engel SS. Efficacy and safety of sitagliptin compared with dapagliflozin in people 65 years oldwith type 2 diabetes and mild renal insufficiency. Diabetes Ther. 2020;11:2419-28.

15. Wang YW, He SJ, Feng X, Cheng J, Luo YT, Tian L, et al. Metformin: a review of its potential indications. Drug Des Devel Ther. 2017;11:2421-9.

Cite this article as: Hazra M. A global analytical molecular pharmacological study of the endocrinological pharmacotherapeutic rationale of anti-diabetic prescriptions appraisal attributes for metformin and sitagliptin, with evaluation of antidiabetic tertiary medical healthcare patient satisfaction. Int J Basic Clin Pharmacol 2021;10:1391-7. 